\title{
Avaliação da autonomia em atividades de leitura e escrita de idosos com baixa visão em intervenção fonoaudiológica: resultados preliminares
}

\author{
Evaluation of autonomy in activities of reading and writing in elderly people with low vision in \\ intervention speech therapy: preliminary results
}

\section{Resumo}

Introdução: A autonomia e a independência, gerando melhora na qualidade de vida, vêm sendo objeto de estudos nos últimos anos, com a crescente taxa de envelhecimento da população, despertando também o interesse da área oftalmológica, fonoaudiológica e de reabilitação. Objetivo: Conhecer as características desta população e avaliar os resultados dos aspectos visuais e de leitura e escrita em idosos com baixa visão, pré e pós-intervenção fonoaudiológica. Método: Realizou-se estudo descritivo e transversal, avaliando-se 23 sujeitos com baixa visão adquirida, atendidos no ambulatório de Visão Subnormal do Hospital de Clínicas da Universidade Estadual de Campinas (VSN-HCUNICAMP). O questionário foi aplicado antes e após a intervenção fonoaudiológica, com duração de três encontros. Resultados: A principal doença ocular encontrada foi o glaucoma (47,8\%). Quinze pacientes $(65,2 \%)$ relataram usar algum tipo de auxílio óptico, dentre eles óculos, lupa, telelupa e régua lupa. Antes de participar dos encontros, $34,8 \%$ dos sujeitos $(n=8)$ relataram não usar a leitura nem a escrita após a perda visual. Depois de participarem dos encontros, 50,0\% $(n=4)$ dos que relataram não mais usar a leitura e a escrita voltaram a utilizá-las. Conclusão: Após a intervenção fonoaudiológica, verificaram-se melhora e frequência de realização das atividades de leitura e escrita. A importância das ações de reabilitação está relacionada à promoção da independência e autonomia para os idosos deficientes visuais.

\section{Abstract}

Introduction: The autonomy and independence, resulting in improved quality of life, have been studied in recent years, due to the increasing rate of population aging, also arousing the interest of ophthalmology, speech therapy and rehabilitation. Objective: To determine the characteristics of this population and evaluate the results in the visual aspects of reading and writing in elderly with low vision, pre and post speech therapy.

Palavras-chave: Qualidade de Vida. Idoso. Baixa Visão. Reabilitação. Fonoaudiologia. Leitura e Escrita.

Programa de Pós-graduação em Ciências Médicas, Faculdade de Ciências Médicas. Universidade Estadual de Campinas. Campinas, SP, Brasil.

2 Departamento de Oftalmologia, Faculdade de Ciências Médicas. Universidade Estadual de Campinas. Campinas, SP, Brasil. 
Methods: We conducted a descriptive and cross-sectional study, evaluating 23 subjects with low vision, from the outpatient clinic of Subnormal Vision of Campinas State University. The questionnaire was administered before and after speech therapy, in three meetings. Results: The most common eye disease was glaucoma (47.8\%). Fifteen patients $(65.2 \%)$ reported using some kind of optical aid, including glasses, magnifying glass, tele-magnifying glass and magnifying glass ruler. Before attending the meetings, $34.8 \%$ of subjects $(\mathrm{n}=8)$ reported no reading or writing after visual loss. After participating in the meetings, $50.0 \%(n=4)$ of those who reported no more reading and writing, used them again. Conclusion: After speech therapy there was improvement and frequency of performing activities of reading and writing. The importance of rehabilitation actions is related to the promotion of independence and autonomy for the visually impaired elderly.

\section{INTRODUCÃO}

Nos países em desenvolvimento, observa-se um envelhecimento populacional acelerado, com incremento da população idosa maior do que nos países desenvolvidos. ${ }^{1}$

O Instituto Brasileiro de Geografia e Estatística (IBGE) ${ }^{2}$ divulgou o resultado do censo de 2010, no qual mostra que os avanços da medicina e as melhorias nas condições gerais de vida da população repercutem no sentido de elevar a média de vida do brasileiro (expectativa de vida ao nascer) de 45,5 anos de idade, em 1940, para 72,7 anos, em 2008 - ou seja, mais 27,2 anos de vida. Segundo a projeção do IBGE, o país continuará galgando anos na vida média de sua população, alcançando em 2050 o patamar de 81,29 anos, basicamente o mesmo nível atual da Islândia (81,80), Hong Kong, China $(82,20)$ e Japão $(82,60)$.

Em 2008, a média de vida para mulheres era 76,6 anos, e para os homens, 69,0 anos - uma diferença de 7,6 anos. Em escala mundial, a esperança de vida ao nascer foi estimada, para 2008 (período 2005-2010), em 67,2 anos e, para 2045-2050, a ONU projeta uma vida média de 75,40 anos.

O processo de envelhecimento no Brasil ocorre rapidamente e num contexto de desigualdades sociais, economia frágil, crescentes níveis de pobreza, com precário acesso aos serviços de saúde e reduzidos recursos financeiros, sem as modificações estruturais que respondam às demandas do novo grupo etário emergente. ${ }^{3,4}$
Key words: Quality of Life. Elderly. Low Vision. Rehabilitation. Speech Pathology. Reading and Writing.
Destaca-se a relevância científica e social de se investigar as condições que interferem no bem-estar na senescência e os fatores associados à qualidade de vida de idosos, no intuito de criar alternativas de intervenção e propor ações e políticas na área da saúde, buscando atender às demandas da população que envelhece. ${ }^{5}$ Consequentemente, muitos questionários foram construídos para mensurar a qualidade de vida, e no campo da oftalmologia não foi diferente. ${ }^{6,7}$

Quando se investiga a qualidade de vida relacionadaàsaúdeemsuamultidimensionalidade, identificam-se os principais aspectos a serem considerados em relação às potencialidades e peculiaridades de saúde e vida do idoso, interferindo no processo saúde-doença. ${ }^{4}$ Dessa forma, avaliar as condições de vida e saúde do idoso permite implementar propostas de intervenção, tanto em programas geriátricos quanto em políticas sociais gerais, no intuito de promover o bem-estar dos que envelhecem. ${ }^{4}$

Adota-se o conceito de deficiência visual na presença de cegueira ou baixa visão. A deficiência visual é o impedimento total ou parcial da capacidade visual, decorrente de uma imperfeição no sistema visual, podendo ser congênita ou adquirida. ${ }^{8}$

Segundo a Organização Mundial da Saúde (OMS), ${ }^{9}$ é considerado cego o indivíduo com acuidade visual entre 3/60 (0,05), no melhor olho e melhor correção óptica possível, até ausência de percepção de luz, ou correspondente perda de campo visual no melhor olho com a melhor correção possível. A baixa visão (visão 
subnormal) corresponde à acuidade visual igual ou menor do que $6 / 18(0,3)$, mas igual ou maior do que $3 / 60(0,05)$ no melhor olho com a melhor correção possível.

A perda da visão, uma das causas mais incapacitantes para o ser humano, tem relação muito estreita com a senilidade. As estruturas oculares sofrem, de forma acumulativa, inúmeros danos metabólicos e ambientais ao longo dos anos. Com isto, as formas mais comuns de patologias oculares são mais frequentes e mais debilitantes nos idosos. A baixa visual é considerada uma queixa frequentemente não referida na população idosa. ${ }^{10}$ As taxas de cegueira e baixa visual aumentam dramaticamente com o aumento da idade. Os problemas visuais em pessoas acima dos 80 anos são de 15 a 30 vezes maiores do que entre pessoas de 40 a 50 anos. ${ }^{9}$

Em relação às principais causas da deficiência visual, a OMS classifica os países e regiões de acordo com o nível de assistência médica estendida à população. Por esse critério, o Brasil poderia ser classificado como país em fase intermediária de desenvolvimento, apresentando como causas da deficiência visual: glaucoma, tracoma, oncocercose, xeroftlamia, catarata e traumatismo. O Brasil, no entanto, apresenta grandes variações regionais. ${ }^{11}$

A deficiência visual é um achado comum em pesquisa envolvendo idosos tanto nos países desenvolvidos quanto em desenvolvimento, devido à crescente incidência de doenças oculares como catarata, degeneração macular relacionada à idade (DMRI), glaucoma e retinopatia diabética. ${ }^{12,13}$ Embora as causas sejam as mesmas, diferentes tipos de intervenções são realizados levando em consideração as características populacionais do Brasil e dos países desenvolvidos. A população idosa desses países geralmente utiliza a leitura e a escrita com mais frequência, exigindo assim um nível compatível de acuidade visual. ${ }^{14}$

Com o avançar da idade, encontram-se, além da presbiopia, a diminuição da sensibilidade aos contrastes, a diminuição da adaptação claroescuro, a demora da recuperação aos ofuscamentos e a diminuição da sensibilidade às cores. ${ }^{15}$
A população idosa com deficiência visual necessita de atenção oftalmológica especializada, incluindo avaliação clínica, aconselhamento e reabilitação para atingir melhoria na qualidade de vida e maior independência. ${ }^{16} \mathrm{~A}$ avaliação clínica e a reabilitação devem envolver a prescrição e adaptação de recursos ópticos e não-ópticos. A utilização de auxílios ópticos e não-ópticos abrange mais do que a melhora da função visual; compreende também o lado emocional do sujeito, tão importante para sua participação efetiva durante o processo de reabilitação, proporcionando melhora na qualidade de vida e inclusão social. ${ }^{17}$

Com o aumento da população idosa no Brasil, esta faixa etária vem ganhando espaço e deixando de ser uma população passiva, para se tornar ativa na sociedade, participante do mundo em sua volta. Percebendo isso, a Organização das Nações Unidas, em 16 de dezembro de 1991, elaborou os "Princípios das Nações Unidas para o Idoso", documento que ressalta a importância do idoso para a sociedade, participando ativamente na formulação e implementação de políticas que afetam seu bem-estar. ${ }^{18}$

A sociedade arca com prejuízos em decorrência da falta de atenção com a saúde visual, representados pela diminuição de produtividade da sua força de trabalho e elevado custo de ações de reabilitação. Acrescem-se a isto consequências psicológicas, sociais e econômicas para a pessoa com deficiência visual, devido às restrições ocupacionais, diminuição de renda, perda de status, autoestima e autoconfiança, sendo estes prejuízos estendidos também à população idosa, que vem modificando, ao longo das últimas décadas, seu papel na sociedade. ${ }^{19}$

Muitos trabalhos têm comprovado a ocorrência de dificuldades no funcionamento emocional em idosos com baixa visão. Burmedi et al., ${ }^{20} \mathrm{em}$ extensa revisão de literatura usando as bases de dados PsyInfo e Medline, língua inglesa e germânica, a partir de 1980, pesquisaram os itens "ajuste emocional" e "relações sociais" em idosos com baixa visão adquirida.

Particularmente em relação à depressão (sentimento de tristeza), várias pesquisas têm 
mostrado que esta ocorre pelo menos duas vezes mais frequentemente que na população idosa geral. ${ }^{21} \mathrm{O}$ ajuste social à perda visual envolve dificuldades no funcionamento emocional, alterações no suporte social e isolamento. As reações emocionais à perda visual são diversas, e a mais óbvia é a depressão, seguida do stress emocional, ansiedade e somatizações. ${ }^{21}$

A Fonoaudiologia ainda é uma ciência nova se comparada a outras profissões da saúde; inicialmente, obteve caráter clínico e evoluiu para ações assistenciais à saúde da população. ${ }^{22}$ Utiliza dados e conclusões de várias outras ciências autônomas e auxiliares, como Biologia, Psicologia, Sociologia, Filosofia, Física, Fonética e Linguística, mas possui unidade própria, sendo um ramo do conhecimento que estuda, de maneira unificada, o fenômeno da comunicação humana. ${ }^{23}$

A comunicação humana abrange o falar, o ouvir, o ler, o escrever e os informes nãoverbais (expressões faciais, gestos, hesitações e o próprio silêncio). Sua qualidade é determinante para autoconfiança, felicidade e segurança, permitindo uma comunicação mais efetiva e fundamental para a saúde do indivíduo. ${ }^{24}$

Em estudos realizados por Monteiro et al., são apresentados resultados em que os sujeitos com deficiência visual deixam de realizar atividades de leitura e escrita após a perda visual. A redução do uso da leitura e da escrita por sujeitos com baixa visão justifica a necessidade de maior ênfase no trabalho com a leitura e a escrita. ${ }^{17} \mathrm{E}$ outra pesquisa ${ }^{25}$ concluiu que há conhecimento do trabalho fonoaudiológico por parte de profissionais da área da saúde e educação que atuam na habilitação/reabilitação de pessoas com deficiência visual, apesar de ainda restrito.

Reconhecendo que o fonoaudiólogo é o profissional que atua na habilitação e reabilitação da comunicação dos sujeitos, seu papel na reabilitação de pessoas com deficiência visual é de extrema importância, pois os sujeitos precisam utilizar meios diferentes daqueles que estavam acostumados para que sua comunicação seja efetiva, possibilitando assim a continuidade de suas relações sociais. ${ }^{17}$

Observando as considerações apresentadas, os objetivos deste estudo foram conhecer as características da população estudada e avaliar a presença ou ausência da melhora na autonomia, independência e atividades de leitura e escrita, considerando os aspectos visuais em idosos com baixa visão adquirida pré e pós-intervenção fonoaudiológica.

\section{MATERIAIS E MÉTODO}

Foi realizado estudo descritivo por meio da utilização do questionário utilizado em tese de livre-docência, ${ }^{21}$ baseado no Low Vison Quality of Life (LVQOL), ${ }^{26}$ traduzido e validado para o português.

Para este estudo, as variáveis escolhidas foram: idade (item 1), causas da baixa visão (estudo de prontuário), escolaridade (item 6), uso de recurso óptico para a leitura e a escrita após a baixa visual (itens 8, 9, 12,16); percepções sobre sua qualidade de vida (item 21).

Trinta e um sujeitos assinaram o Termo de Consentimento Livre e Esclarecido e compareceram ao primeiro encontro. No segundo encontro, 28 sujeitos compareceram. No terceiro, somente 23. Deste modo, a amostra foi constituída por 23 sujeitos que estavam dentro dos critérios de inclusão: idosos (60 anos ou mais, segundo o Estatuto do Idoso); com baixa visão adquirida, atendidos no ambulatório de Visão Subnormal do Hospital de Clínicas da Universidade Estadual de Campinas (VSN-HC-UNICAMP) e que participaram dos três encontros mensais (em abril, maio e junho de 2011).

Os encontros foram realizados em grupos previamente agendados, com duração de 50 
minutos. Os pacientes eram colocados em círculo e não houve participação de nenhum cuidador e/ou parente. A cada encontro, um tema diferente era abordado pela pesquisadora: atividades de vida diária, auxílios ópticos e não-ópticos (necessidade, prescrição, uso, frequência do uso e melhora da visão), e por último, leitura e escrita. Durante os encontros, a pesquisadora disponibilizava novos recursos para os participantes realizarem suas atividades diárias, como também sanava dúvidas em relação aos auxílios ópticos e não-ópticos e de leitura e escrita. Exercícios e treinamento eram realizados para demonstrar aos pacientes como eles deveriam ser feitos.

Os pacientes eram encorajados a discutirem os temas e também dividirem suas próprias experiências. Ao final do terceiro encontro, o questionário foi reaplicado para comparação das respostas. O questionário foi aplicado pela própria pesquisadora e sua leitura foi feita de forma fidedigna. Também foi realizado estudo de prontuário para pesquisa de dados médicos, como hipótese diagnóstica; acuidade visual para longe e para perto, com ou sem uso de auxílios; e auxílios ópticos prescritos. O programa Epi-Info (versão 6.0) foi utilizado para analisar os dados estatisticamente.
O projeto foi aprovado pelo Comitê de Ética em Pesquisa da Faculdade de Ciências Médicas da Universidade Estadual de Campinas, sob o ${ }^{\circ}$ 1.041/2010.

\section{RESULTADOS}

A amostra foi composta por 23 sujeitos, com faixa etária compreendida entre 60 e 91 anos e média de idade de 71 anos. Em relação à escolaridade, $4,3 \%$ dos sujeitos nunca estudaram, $78,2 \%$ têm ensino fundamental incompleto, $4,3 \%$ têm ensino médio incompleto e $13,2 \%$ têm ensino superior completo.

A principal causa da baixa visão encontrada nos sujeitos foi o glaucoma (47,8\%), segundo a tabela 1. Oito sujeitos $(34,8 \%)$ relataram não fazer uso de nenhum auxilio óptico. Destes, quatro $(50,0 \%)$ relataram não se adaptar aos auxílios. Três sujeitos $(37,5 \%)$ não conseguiram adquirir o auxilio; e um (12,5\%) não achava necessário seu uso. Quinze sujeitos (65,2\%) relataram usar algum tipo de auxílio óptico; destes, $14(93,3 \%)$ relataram fazer uso de óculos. Os motivos do não-uso dos auxílios ópticos e os tipos dos auxílios ópticos usados são apresentados na tabela 2 .

Tabela 1 - Causas da baixa visão em sujeitos idosos atendidos no VSN-HC-UNICAMP. Campinas, SP, 2011. $(n=23)$

\begin{tabular}{lcc}
\hline Causas* $^{*}$ & $\mathrm{f}$ & $\%$ \\
\hline Glaucoma & 11 & 47,8 \\
Catarata & 7 & 30,4 \\
Degeneração macular relacionada à idade & 2 & 8,6 \\
Retinopatia diabética & 2 & 8,6 \\
Outras causas & 5 & 21,5 \\
\hline
\end{tabular}

*Múltiplas causas em quatro pacientes. 
Tabela 2 - Uso, motivo do não-uso e tipo de auxílios ópticos em sujeitos idosos atendidos no VSNHC-UNICAMP. Campinas, SP, 2011. ( $\mathrm{n}=23$ )

\begin{tabular}{lccc}
\hline Uso de auxilio óptico & $\mathrm{f}$ & $\%$ & \\
\hline Declaram fazer uso & 15 & 65,2 & \\
Declaram não fazer uso & 8 & 34,8 & \\
\hline Motivos para o não-uso & & & $\mathrm{n}=8$ \\
\hline Sem adaptação & 4 & 50,0 & \\
Sem aquisição & 2 & 25,0 & \\
Sem necessidade & 2 & 25,0 & \\
\hline Tipos de auxilio ópticos usados* & $\mathrm{f}$ & $\%$ & $\mathrm{n}=15$ \\
\hline Óculos & 14 & 93,3 & \\
Lupa & 7 & 46,6 & \\
Telelupa & 1 & 6,6 & \\
Régua lupa & 1 & 6,6 & \\
\hline
\end{tabular}

* Uso de mais de um tipo de auxilio óptico por sete pacientes.

Antes de participarem dos encontros, 34,8\% dos sujeitos (8) relataram não usar a leitura nem a escrita após a perda visual. Dos 65,3\% ( $\mathrm{n}=15)$ que ainda as utilizavam, em relação à leitura, $100 \%$ preferem ler letras grandes e $66,6 \%(n=10)$ utilizam auxílios ópticos para essa atividade. Em relação à escrita, 86,6\% (n=13) não utilizam nenhum tipo de auxilio óptico para esta atividade. E 100\% (dos 13) têm dificuldade para ler a própria escrita, o que leva à não-realização desta atividade.

Depois de participarem dos encontros, $50,0 \%(n=4)$ dos que relataram não mais usar a leitura e a escrita voltaram a utilizá-las com a ajuda dos auxílios ópticos prescritos e com as orientações recebidas durante os encontros, havendo melhora em sua leitura, especialmente na de perto. Ao final dos encontros, 19 sujeitos $(82,6 \%)$, dos que continuavam a utilizar a leitura após a baixa visual e dos que voltaram a usála depois de participarem do estudo, relataram melhoras relacionadas à leitura (tabela 3). Em relação à escrita, 13 sujeitos (56,2\%), do total de participantes do estudo relataram começar a utilizá-la para se comunicar com outras pessoas.

Tabela 3 - Melhoras relacionadas à leitura após intervenção fonoaudiológica em sujeitos idosos atendidos no VSN-HC-UNICAMP. Campinas, SP, 2011. (n=23)

\begin{tabular}{lcc}
\hline Melhoras* & $\mathrm{f}$ & $\%$ \\
\hline Leitura de perto & 19 & 100 \\
Leitura de sua própria letra & 13 & 68,4 \\
Leitura de longe & 6 & 31,5 \\
Leitura por mais tempo & 6 & 31,5 \\
\hline
\end{tabular}

*Mais de um tipo de melhora para oito sujeitos. 
Sobre a percepção dos sentimentos em relação ao problema oftalmológico, apresenta-se a tabela 4: $69,5 \%$ dos sujeitos $(n=15)$ que participaram do estudo relataram que se sentiam tristes após a baixa visual antes de participarem da intervenção com a fonoaudióloga, sendo que desses 15, dez sujeitos $(66,6 \%)$ relataram se sentirem assim devido a seu problema visual. Após a intervenção fonoaudiológica, $52,1 \%(\mathrm{n}=12)$ relataram que ainda continuam tristes após a intervenção fonoaudiológica.

Tabela 4 - Percepções dos sentimentos em relação ao problema oftalmológico, pré e pósintervenção fonoaudiológica. Campinas, SP, 2011. ( $\mathrm{n}=23$ )

\begin{tabular}{|c|c|c|}
\hline Costumam se sentir triste & & \\
\hline Antes da intervenção & f & $\%$ \\
\hline Sim & 15 & 69,5 \\
\hline Não & 8 & 30,5 \\
\hline \multicolumn{3}{|l|}{ Após a intervenção } \\
\hline $\operatorname{Sim}$ & 12 & 52,1 \\
\hline Não & 11 & 47,9 \\
\hline \multicolumn{3}{|l|}{ Motivos para se sentir triste } \\
\hline Antes da intervenção $\quad \mathrm{n}=15$ & $\mathrm{f}$ & $\%$ \\
\hline Porque não enxerga bem & 10 & 66,6 \\
\hline Por outros motivos & 1 & 6,6 \\
\hline Por causa da vista e por outras razões & 4 & 24,8 \\
\hline Após a intervenção $\quad \mathrm{n}=12$ & $\mathrm{f}$ & $\%$ \\
\hline Porque não enxerga bem & 8 & 66,6 \\
\hline Por outros motivos & 1 & 8,3 \\
\hline Por causa da vista e por outras razões & 3 & 25,1 \\
\hline
\end{tabular}

\section{DISCUSSÃO}

O ensino fundamental incompleto $(78,2 \%)$ foi o grau de escolaridade que se apresentou em maior proporção, assim como no estudo de Carvalho. ${ }^{14}$ Esse dado apresenta a baixa escolaridade dos sujeitos participantes da pesquisa, podendo ser uma das causas da não-utilização da leitura e da escrita após a perda visual, assim como podese especular que o uso da leitura e da escrita apresentava-se pouco mesmo antes da doença ocular, sendo a baixa visual um agravante para a não-utilização.
Neste estudo, a principal causa da baixa visão foi o glaucoma, ao contrário do que mostra grande parte da literatura brasileira e internacional. A degeneração macular relacionada à idade, que é a principal causa da baixa visão em pacientes com mais de 65 anos, ${ }^{27-}$ ${ }^{29}$ foi citada somente algumas vezes.

A sociedade arca com prejuízos elevados em decorrência da falta de atenção com a saúde visual, representados pela diminuição de produtividade da sua força de trabalho e o elevado custo de ações de reabilitação. Acrescem-se a isto consequências 
psicológicas, sociais e econômicas para o sujeito com deficiência visual, devido a restrições ocupacionais, diminuição da renda, perda de status, autoestima e autoconfiança. ${ }^{19}$ Assim, é essencial que estudos epidemiológicos no campo oftalmológico continuem sendo realizados para que se possa ter a real perspectiva de doenças oculares que afetam a população idosa, propondo ações preventivas e reabilitacionais para essas doenças, com foco nas necessidades apresentadas por esses sujeitos. ${ }^{27}$

O uso do recurso óptico foi relatado pela maioria dos sujeitos deste estudo ( $\mathrm{n}=15,65,2 \%$ ). Alguns necessitaram de mais de um auxílio óptico para melhorar o uso do seu resíduo visual $(n=7,46,6 \%)$. Esse recurso tem como objetivo melhorar a visão quantitativamente, ajudando a desempenhar as atividades de vida diária. Para o sucesso da prescrição de um auxílio óptico, a avaliação por serviço especializado composto por equipe interdisciplinar é necessária, levandose em conta também a acuidade visual, a sensibilidade aos contrastes e as necessidades do paciente, verificando-se em quais situações cotidianas a visão está fazendo falta. O uso desses auxílios especiais é de extrema importância, pois leva a uma melhoria na autonomia e na qualidade de vida desses sujeitos ${ }^{14,29}$ (tabela 2).

Em estudo realizado em Campinas, ${ }^{17}$ a maioria dos sujeitos $(60,0 \%)$ declarou fazer uso de algum tipo de auxílio óptico na realização das atividades de longe e de perto. Sacks ${ }^{29}$ afirmou que o uso de auxílios ópticos pode aumentar a autoestima de sujeitos com baixa visão. Entre os benefícios do uso desses auxílios, ressaltam-se: o senso de independência (quando este sujeito consegue ter acesso à leitura de materiais impressos no ambiente diário, ele se torna independente); o senso de responsabilidade (ao adquirir a informação visual real, ele percebe seu potencial e se sente responsável e seguro para enxergar em outras situações); melhora a identificação do ambiente; o senso de competência (porque o sujeito tem controle visual sobre o ambiente) e o sentimento de maior prazer pela qualidade visual.

A prescrição de auxílios ópticos de acordo com o nível de visão apresentado de forma geral melhora o desempenho visual, dependendo do tempo de aquisição da doença oftalmológica, do nível de acuidade visual e das crenças em relação à saúde ocular. Estudo realizado na Inglaterra evidencia o acompanhamento, por seis meses consecutivos, de 168 pacientes com baixa visão que tiveram prescrição de auxílio óptico associados à orientação do uso de auxílios não-ópticos. No que se refere à atividade de leitura, melhoraram significativamente o grau de desempenho, sendo que $88,0 \%$ dos sujeitos começaram a ler letras impressas em jornais depois do tratamento mencionado. ${ }^{30}$

Em relação ao motivo do não-uso de auxílios ópticos, a maioria $(50,0 \%)$ declarou que não o faz pela não adaptação ao auxílio. Após a escolha do auxílio óptico, a adaptação é muito importante, pois se for realizada de forma correta, o auxílio será utilizado corretamente; caso contrário, ficará numa gaveta e não será usado. ${ }^{31}$

O auxilio óptico mais citado pelos sujeitos da pesquisa foi o óculos (93,3\%), concordando com outros estudos..$^{14,17,32}$ Os óculos aparecem com frequência tão alta devido a sua comodidade. Colocando-o no rosto, o sujeito necessita então encontrar a melhor posição para enxergar, porém deixa as mãos livres para realizar as atividades. ${ }^{33}$

De maneira geral, as experiências nas atividades de vida diária mostram que a maioria dos sujeitos com deficiência visual manifesta dificuldades ante a prática visual das atividades de vida diária/atividades de vida prática (AVD/ AVP). O uso de auxílios ópticos mostra-se eficaz na realização dessas atividades, melhorando o desempenho visual dos sujeitos.

Para facilitar e promover o uso frequente dos auxílios ópticos, os sujeitos devem usá-los sempre em tarefas que sejam de seu interesse, os períodos devem ser seriados e curtos, evitando assim, cansaço físico e visual. ${ }^{16} \mathrm{O}$ sujeito com baixa visão só usará o auxílio óptico se este corresponder às suas possibilidades e a realidade de sua vida. $O$ processo de potencialização no uso do auxílio óptico inclui ajuda ao paciente no domínio da técnica, na escolha do auxílio óptico mais adequado, na prática das atividades 
de seu interesse, como escrita, leitura e atividade de vida diária.

O número restrito de sujeitos $(34,8 \%)$ que ainda utilizavam a leitura e a escrita após a baixa visual caracteriza a dificuldade encontrada em realizar as atividades após a doença ocular. Desta forma, a prescrição de auxílios ópticos e a reabilitação visual mostram-se de extrema importância para que os sujeitos voltem a realizar tais atividades, devolvendo sua autonomia e melhorando sua qualidade de vida.

As dificuldades poderiam ser minimizadas pela utilização dos auxílios ópticos e não-ópticos e adaptação adequada, visto que um dos principais objetivos de um programa de reabilitação para pessoas com baixa visão é fazer com que os sujeitos melhorem a eficiência do resíduo visual, sentindo-se confortáveis com seu auxílio. ${ }^{34} \mathrm{~A}$ participação em um programa de reabilitação pode minimizar os aspectos negativos do uso da visão residual: a dificuldade para enxergar e o cansaço visual. ${ }^{35}$

Dentro do contexto de adaptação dos auxílios ópticos frente à leitura, é importante a elaboração de um processo gradativo que minimize as dificuldades a ela relacionadas, ${ }^{36}$ assim favorecendo o uso contínuo da leitura no cotidiano das pessoas com baixa visão.

Em relação à escrita, 86,6\% dos sujeitos relataram não usar nenhum tipo de auxilio óptico para realizar esta atividade, mas sujeitos com baixa visão podem escrever sem usar nenhum tipo de auxilio óptico. Na maioria das vezes, entretanto, eles não conseguem ler o que escreveram e acabam por deixar de usar a escrita. Isso demonstra também a importância de utilizar o auxilio óptico para a atividade de escrita.

A escrita para os adultos é algo tão extremamente envolto no cotidiano que, na maioria das vezes, não nos damos conta da importância e utilidade à nossa manutenção e sobrevivência. Em um mundo constituído pela palavra escrita, todavia, não se tem o hábito de refletir sobre as dificuldades encontradas por uma pessoa que apresenta dificuldades visuais e que é diariamente exposta ao conhecimento de ler e escrever. ${ }^{17}$ A leitura e a escrita caminham juntas na comunicação humana, e são de extrema importância para promover a independência e socialização dos sujeitos.

O meio mais importante de aquisição de conhecimentos, mesmo em tempos de internet e do mundo virtual, ainda é a linguagem escrita. Saber ler e escrever é requisito essencial para que uma pessoa participe efetivamente da sociedade em que vive e seja um cidadão consciente.

A leitura feita por outras pessoas faz com que a pessoa com deficiência visual seja dependente de alguém para realizar esta atividade. Deve-se levar em consideração também que cada pessoa realiza a leitura de forma diferente, tanto no tempo necessário para realizá-la, como nas inferências feitas, que são individuais, dando sentido e contexto para o que está sendo lido. ${ }^{37}$

Após os encontros, os pacientes relataram melhora nas atividades de leitura e escrita, como a leitura para perto $(100 \%)$ e a leitura da própria letra $(68,4 \%)$, como também o uso da escrita para se comunicar com outras pessoas $(56,2 \%)$.

Considerando a leitura um importante fator de inclusão social, econômico e cultural, faz-se necessária uma ênfase na atividade de leitura durante o processo de reabilitação. ${ }^{17} \mathrm{~A}$ reaprendizagem ou utilização da leitura e da escrita no processo de reabilitação visual pode vir a ser uma motivação maior da pessoa para usufruíla com mais prazer e frequência. Este recurso visaria como resultado uma maior autonomia por parte das pessoas com deficiência visual. ${ }^{17}$

Em relação à percepção de seus sentimentos após o problema oftalmológico, a maior parte das pessoas $(69,5 \%)$ relatou que se sentia triste após a baixa visual antes da intervenção fonoaudiológica. Após a intervenção, a maioria $(52,1 \%)$ também relatou que se sentia triste, porém esse número diminuiu (de 15 para 12 sujeitos). Estudo realizado por Carvalho ${ }^{22}$ também encontrou percentagem significativa de sujeitos que apresentavam percepções negativas em relação a seus sentimentos após a baixa 
visual. O principal motivo citado pelos sujeitos que declararam se sentir tristes após a baixa visual, tanto antes como após a intervenção fonoaudiológica, foi justamente a baixa visual.

$\mathrm{Na}$ reabilitação de pessoas com baixa visão, a Fonoaudiologia tem o papel de agregar o uso do recurso óptico à leitura e à escrita de forma efetiva, mostrando ao indivíduo que, utilizando diferentes estratégias, a linguagem escrita pode e deve fazer parte de seu cotidiano, auxiliando-o a comunicar-se com as pessoas de seu convívio por meio da leitura e da escrita. Segundo estudo de Montilha et al., ${ }^{8}$ a comunicação escrita por meio da leitura e da escrita, propriamente dita, reflete $56,0 \%$ e $52,0 \%$ das limitações, respectivamente, segundo opinião de sua população.

O presente estudo auxilia na afirmação da importância das atividades de leitura e escrita dentro de um processo de reabilitação, podendo ser realizado pelo fonoaudiólogo que integre a equipe interdisciplinar, desde que o profissional se aproprie disso. A formação da maioria dos fonoaudiólogos do país não contempla o conhecimento sobre a deficiência visual, por isso sua prática nesse campo tem sido muito restrita. Desta forma, percebe-se que o fonoaudiólogo é um profissional a mais a se inserir em uma equipe interdisciplinar, tanto na habilitação como na reabilitação das pessoas com deficiência visual, empregando seus conhecimentos específicos de forma a auxiliar os demais membros da equipe e os sujeitos a realizarem as atividades de vida diária com ênfase na leitura e na escrita.

\section{CONCLUSÃO}

A população deste estudo tem média de idade de 71 anos e baixa escolaridade. A principal causa da baixa visão encontrada foi o glaucoma e a maioria dos sujeitos faz uso de algum auxilio óptico. Após a intervenção fonoaudiológica, metade dos sujeitos que não utilizavam auxílio óptico voltou a fazê-lo.

Os sujeitos que ainda utilizavam a leitura após a baixa visual relataram que sua leitura melhorou após a reabilitação. Os participantes do estudo voltaram a utilizar a escrita, principalmente como forma de comunicação.

A qualidade de vida dos idosos é diretamente afetada por seu problema visual. Com este estudo, é possível afirmar que a prescrição de auxílios ópticos, seu treinamento e a reabilitação visual são fatores de extrema importância para sua autonomia e independência, influenciando na qualidade de vida dessa população.

\section{REFERÊNCIAS}

1. Organizacion Panamericana de La Salud. Condiciones de salud en las Américas. Washington: OPS; 1994.

2. Instituto Brasileiro de Geografia e Estatística. Censo Demográfico 2010. [acesso em 2 dez 2012]. Disponível em: http://www.ibge.gov.br/home/ estatistica/populacao/censo2010/default.shtm.

3. Chaimowick F. A saúde dos idosos brasileiros às vésperas do século XXI: problemas, projeções e alternativas. Rev Saude Publica 1997;31(2):184-200.

4. Pereira RJ, Cotta RMM, Franchescini SCC, Ribeiro RCL, Sampaio RF, Priore SE, et al. Contribuição dos domínios físico, social, psicológico e ambiental para a qualidade de vida global de idosos. Rev Psiquiat Rio Gd Sul 2006;28(1):37-8.
5. Fleck MPA, Chachamovich E, Trentini CM. Projeto WHOQOL-OLD: método e resultados de grupos focais no Brasil. Rev Saude Publica 2003;37(6):793-9.

6. Zou H, Zhang X, Xu, X, Bai L, Wolffsohn JS. Development and psychometric tests of the Chineseversion Low Vision Quality of Life Questionnaire. Qual Life Res 2005;14(6):1633-9.

7. Boer MR, Terwee CB, Vet HC, Moll AC, VolkerDieben HJ, Van Rens GH. Evaluation of crosssectional and longitudinal construct validity of two vision-related quality of life questionnaires: the LVQOL and VCM1. Qual Life Res 2006;15(2):233-48.

8. Montilha RCI, Temporini, ER, Nobre, MIR, Gasparetto, MERF, Kara-José, N. Utilização de 
recursos ópticos e equipamentos por escolares com deficiência visual. Arq Bras Oftalmol 2006;69(2):207-11.

9. World Health Organization (WHO) - Global date on blindness. (Bulletin of the World Health Organization). Genivè. 1995; 73(1): 115-120.

10. Thylefors B, Négrel AD, Pararajasegaram R, Dadzie KY. Global date on blindness. Bull World Health Organ 1995;73(1):115-21.

11. Keane EM, O'Connor M, Coakley D, Walsh JB. Eye screening in the elderly. Ir Med J 1997;90(4):141-2.

12. Rodrigues MLV. Prevenção de perdas visuais. Rev Hosp Clin Fac Med Rib Preto Univ São Paulo 1997;30(1):84-9.

13. Brenner MH, Curbow B, Javitt JC, Legro MW, Sommer A. Vision change and quality of life in the elderly. Response to cataract surgery and treatment of other chronic ocular conditions. Arch Ophthalmol 1993;111(5):680-5.

14. Human L, Wu SY, Connell AM, Schachat A, Nemesure B, Hennis A, et al. Prevalence and causes of visual impairment in The Barbados Eye Study. Ophthalmol 2001;108(10):1751-6.

15. Carvalho KM, Monteiro GB, Isaac CR, Shiroma LO, Amaral MS. Causes of low vision and use of optical aids in the elderly. Rev Hosp Clin Fac Med Sao Paulo 2004;59(4):157-60.

16. Romani FA. Prevalência de transtornos oculares na população de idosos residentes na cidade de Veranópolis, RS, Brasil. Arq Bras Oftalmol 2005;68(5):649-55.

17. Carvalho KMM. Recursos para visão subnormal. Arq Bras Oftalmol 1997;60(3):317-9.

18. Monteiro MBM, Montilha RCI, Gasparetto MERF. Atenção fonoaudiológica e a linguagem escrita de pessoas com baixa visão: estudo exploratório. Rev Bras Ed Esp Marília 2011;17(1):121-36.

19. Envelhecimento \& Saúde. Boletim Instituto de Saúde 2009;47. [acesso em ?? mes 20??]. Disponível em: www.isaude.sp.gov.br

20. Kara-Jose N, Temporini ER. Cirurgia de catarata: o porquê dos excluídos. Rev Panam Salud Publica 1999;6(4):242-8.

21. Burmedi D, Becker S, Heyl V, Wahl H-W, Himmelsbach I. Emotional and social consequences of age-related low vision. Vis Impair Research 2002;4(1):47-71.

22. Carvalho KMM. Baixa visão em idosos: causas, estado funcional, percepções de limitações e reabilitação visual em unidade hospitalar universitária.
Campinas. Tese [Livre-Docência em Ciências

Médicas] - Universidade Estadual de Campinas; 2007.

23. Conselho Regional de Fonoaudiologia: 2a Região. Quatro décadas de evolução da fonoaudiologia. Rev Fonoaudiol 2002;44:7-8.

24. Amorim A. Fonoaudiologia Geral. São Paulo: Ed. Pioneira; 1972.

25. Andrade CRF. Fonoaudiologia preventiva. São Paulo: Lovise; 1996.

26. Monteiro MMB, Montilha RCI. Intervenção fonoaudiológica e deficiência visual: percepções de profissionais de equipe interdisciplinar. Medicina (Ribeirão Preto) 2010;43(1):11-9.

27. Wolffsohn JS, Cochrane AL. Design of the low vision quality-of-life questionnaire (LVQOL) and measuring the outcome of low-vision rehabilitation. Am J Ophthalmol 2000;130(6):793-802.

28. Subramanian A, Pardhan S. Repeatability of reading ability indices in subjects with impaired vision. Invest Ophthalmol Vis Sci 2009;50(8):3643-7.

29. Foran S, Wang JJ, Mitchell P. Causes of visual impairment in two older population cross-sections: the Blue Mountains Eye Study. Ophthalmic Epidemiol 2003;10(4):215-25.

30. Brody BL, Gamst AC, Williams RA, Smith AR, Lau PW, Dolnak D, et al. Depression, visual acuity, comorbidity, and disability associated with agerelated macular degeneration. Ophthalmology 2001;108(10):1893-900; discussion 1900-1

31. Araujo LF, Coutinho MPL, Carvalho VAML. Representações sociais da velhice entre idosos que participaram de grupos de convivência. Psicol Ciênc e Prof 2005;25(1):118-31.

32. Burman-Lindelow P. Magnificação e auxílios ópticos em baixa visão. In: Veitzman, S. Visão subnormal. Rio de Janeiro: Cultura Médica; 2000. p. 111-22.

33. Freitas CC, Monteiro GB, Gasparetto MERF, Carvalho KMM. Assistive technology applied to education of students with visual impairment. Revista Panamericana de Salud Pública (Impresa) / Pan American Journal of Public Health (Impressa). 2009; 26(2): 148-152.

34. Alves CC, Monteiro GB, Rabello S, Gasparetto ME, Carvalho KM. Assistive technology applied to education of students with visual impairment. Rev Panam de Salud Publica 2009;26(2):148-52.

35. Sacks SZ. Psychological and social implications of low vision. In: Corn AL, Koenig AJ. Foundation of low vision: clinical and functional perspective. New York: American Foundation for The Blind Press 1966. p. 26-42. 
36. Margrain TH. Helping blind and partially sighted people to read: the effectiveness of low vision aids. $\mathrm{Br}$ J Ophthalmol 2000;84(8):919-21.
37. Lucas MB, Leal DB, Tavares SS, Barros EA, Aranha ST. Condutas reabilitacionais em pacientes com baixa visão. Arq Bras Oftalmol 2003; 66(1):77-82.

Recebido: 09/1/2012

Revisado: 30/5/2012

Aprovado: 17/11/2012 